\title{
Visible implant elastomer (VIE) success in early larval stages of a tropical amphibian species
}

\author{
Chloe A Fouilloux ${ }^{\text {Corresp., }}{ }^{,}$, Guillermo Garcia-Costoya ${ }^{1}$, Bibiana Rojas ${ }^{1}$ \\ ${ }^{1}$ Department of Biology and Environmental Science, University of Jyväskylä, Jyväskylä, Finland \\ Corresponding Author: Chloe A Fouilloux \\ Email address: chloe.a.fouilloux@jyu.fi
}

Animals are often difficult to distinguish at an individual level, and being able to identify individuals can be crucial in ecological or behavioral studies. In response to this challenge, biologists have developed a range of marking (tattoos, brands, toe-clips) and tagging (banding, collars, PIT, VIA, VIE) methods to identify individuals and cohorts. Animals with complex life cycles are notoriously hard to mark because of the distortion or loss of the tag across metamorphosis. In amphibians, few studies have attempted larval tagging and none have been conducted on a tropical species. Here, we present the first successful account of VIE tagging in early larval stages (Gosner stage 25) of the dyeing poison frog (Dendrobates tinctorius) coupled with a novel anaesthetic (2-PHE) application for tadpoles that does not require buffering. Mean weight of individuals at time of tagging was $0.12 \mathrm{~g}$, which is the smallest and developmentally youngest anuran larvae tagged to date. We report $81 \%$ tag detection over the first month of development, as well as the persistence of tags across metamorphosis in this species. Cumulative tag retention versus tag observation differed by approximately $15 \%$ across larval development demonstrating that "lost" tags can be found later in development. Tagging had no effect on tadpole growth rate or survival. Successful application of VIE tags on D. tinctorius tadpoles introduces a new method that can be applied to better understand early life development and dispersal in various tropical species. 


\section{VISIBLE IMPLANT ELASTOMER (VIE) SUCCESS IN EARLY}

\section{LARVAL STAGES OF A TROPICAL AMPHIBIAN SPECIES}

3

4 Chloe Fouilloux ${ }^{* 1}$, Guillermo Garcia-Costoya ${ }^{1}$, and Bibiana Rojas ${ }^{1}$

$5 \quad{ }^{1}$ Department of Biology and Environmental Science, University of Jyväskylä, Finland

6 Corresponding Author:

7 Chloe Fouilloux*1

8

9 Email address: chloe.a.fouilloux@.jyu.fi

10

11

12

13

14

15

16

17

18

19

20

21

22

23

24

25

26

27

28

29

30

31

32

33

34

35

36 
37

38

39

40

\section{1}

42

43

44

45 46

\section{Abstract}

Animals are often difficult to distinguish at an individual level, and being able to identify individuals can be crucial in ecological or behavioral studies. In response to this challenge, biologists have developed a range of marking (tattoos, brands, toe-clips) and tagging (banding, collars, PIT, VIA, VIE) methods to identify individuals and cohorts. Animals with complex life cycles are notoriously hard to mark because of the distortion or loss of the tag across metamorphosis. In amphibians, few studies have attempted larval tagging and none have been conducted on a tropical species. Here, we present the first successful account of VIE tagging in early larval stages (Gosner stage 25) of the dyeing poison frog (Dendrobates tinctorius) coupled with a novel anaesthetic (2-PHE) application for tadpoles that does not require buffering. Mean weight of individuals at time of tagging was $0.12 \mathrm{~g}$, which is the smallest and developmentally youngest anuran larvae tagged to date. We report $81 \%$ tag detection over the first month of development, as well as the persistence of tags across metamorphosis in this species. Cumulative tag retention versus tag observation differed by approximately $15 \%$ across larval development demonstrating that "lost" tags can be found later in development. Tagging had no effect on tadpole growth rate or survival. Successful application of VIE tags on D. tinctorius tadpoles introduces a new method that can be applied to better understand early life development and dispersal in various tropical species.

\section{Introduction}

Distinguishing individuals within a population is often key in deciphering animal behavior, lifehistory traits, and ecological dynamics. Animal identification has applications in understanding parental care (Ménard et al., 2001), migration dynamics (Matthews et al., 2011; Fuller et al., 2008), adaptations to environmental pressures (Warne and Crespi, 2015; Gordon et al., 2009), and even fecundity (Martin, 1995). Studies across the animal kingdom have developed methods that vary both in invasiveness and success (guppies: Croft et al., 2003; Gordon et al. 2009; 
69 salamanders: Osbourn et al., 2011; turtles: Fuller et al. 2008; birds: Martin, 1995; dolphins:

70 Defan et al., 1990; bears: Diefenbach \& Alt, 1998) to allow researchers to differentiate between

71 individuals within groups. If visual differentiation is not an obvious option, physical

72 manipulation (e.g. toe clips, tattoos; Perret \& Joly, 2002; Phillott et al., 2007) and tagging (e.g.

73 passive integrated transponder (PIT), Perret \& Joly, 2002; visible implant alphanumeric (VIA),

74 Caballero-Gini et al. 2019; visible implant elastomer (VIE), Brannelly et al. 2013; ear tags,

75 Diefenbach and Alt, 1998; banding, Martin, 1995; and collars, Gese, 2001) have been the most

76 commonly used methods implemented in mark-recapture studies.

77

Differentiating individuals is important when there is a lot of intrapopulation variation in behavior, and is becoming especially relevant as we begin to see individuals adapt to new challenges onset by the effects of global warming, habitat fragmentation, and human interactions. However, long-term mark-based studies have not often been applied across ontogenetic stages of animals with complex life cycles, as the physical transformations induced with metamorphosis and growth generally entail the loss or distortion of the mark. In amphibians, there have been a range of successful tagging methods both in adult and larval stages, but the diversity of larval tagging studies has been limited to common temperate species (e.g., Campbell Grant, 2008; Courtois et al., 2013), and very few studies have been able to create a methodology that spans the animal's entire life cycle (Bailey, 2004; Bainbridge et al., 2015; Caballero-Gini et al., 2019; Campbell Grant, 2008; McHarry et al., 2018).

Understanding the dispersion dynamics and survival of amphibians from aquatic to terrestrial habitats makes developmentally early larval tagging especially interesting. Tagging in amphibian tadpoles could be used to understand how environmental stress affects individual development in group conditions (e.g. Dendropsophus ebracattus (Touchon et al., 2011), Agalychnis callidryas (Gonzalez et al., 2011), Triturus alpestris (Denoel \& Joly, 2000)) or could be used to investigate the dynamics of aggressive displays between tadpoles (e.g. Ambystoma tigrinum nebulosum (Pfennig et al., 1991), Rana utricularia (Faragher \& Jaeger, 1998), Oophaga pumilio (Dugas et al., 2016)). Many species of Neotropical poison frogs have parental care where recently hatched tadpoles are transported from terrestrial sites to arboreal pools (Pašukonis et al., 2019; Ringler et al., 2013; Schulte \& Mayer, 2017; Summers \& Tumulty, 2013). Tadpole tagging could provide a 
100 quick and reliable method of following individuals across development, understanding

101 relatedness within pools, and observing tadpole behavior and parental care in the field. In this

102 study, we mark the larvae of Dendrobates tinctorius, a neotropical species of poison frog whose

103 tadpoles develop in ephemeral pools of water.

Larval anuran tagging has been limited with respect to both developmental stage and weight. Most of the inter-stage larval tagging to date has been done beyond the point of the onset of hind leg development (Andis, 2018 (Gosner stage 30); Bainbridge et al., 2015 (Gosner stage 36-38); Gosner 1960). At this stage, D. tinctorius tadpoles are typically at least a month old, meaning they have already been transported by their fathers and have long since been subject to both predation risk and aggression by conspecifics (CF personal observation; Rojas, 2014, 2015;

111 Rojas \& Pašukonis, 2019). Therefore, in order to obtain more valuable life history information,

112 tags need to be applied earlier in development.

114 To our knowledge, the developmentally earliest tagging study applied VIA/VIE tags around

115 Gosner stage 25 (Courtois et al., 2013), but its application was limited to large temperate

116 tadpoles (average weight around $1.5 \mathrm{~g}$ ) that could be manipulated in the field without anesthesia.

117 In this study we use 2-phenoxyethanol (2-PHE), an anesthetic that does not need to be buffered

118 and can be stored at room temperature, making it field appropriate (Acme-Hardestry, 2013;

119 National Center for Biotechnology, 2020). 2-PHE has been used on newts (Perret and Joly

120 2002), fishes (Toni et al., 2015), and adult frogs (Eggert et al., 1999) for anesthetic purposes, but

121 has been largely overlooked for larval application. In most amphibian research MS-222 has been

122 used, popularized perhaps because of its use in amphibian medicine (Mitchell, 2009; Vrskova

123 and Modra, 2012). Yet, MS-222 needs to be buffered and has been found to increase cortisol

124 concentrations (an indicator of stress) (Coyle et al., 2004). In comparison, studies using 2-PHE

125 on fish found that this anesthetic prevented the induction of stress pathways during stressful

126 procedures (Toni et al., 2015). Further, 2-PHE has a large safety margin for applied doses and

127 can be easily acquired through common compound manufacturers (Matthews and Varga, 2012).

128

129 In this study, we apply VIE tags to the smallest and developmentally earliest stages of larval 130 anurans recorded to date. We follow growth rate and tag success across larval development, and 
131 discuss potential field applications in order to better understand the dispersion dynamics and

132 behavior of protected frogs.

133

134

135

136

137

138

139

140

141

142

143

144

145

146

147

148

149

150

151

152

153

154

155

156

157

158

159

160

161

\section{Materials \& Methods}

Study organism - Dendrobates tinctorius is a neotropical poison frog that transports their recently hatched larvae to ephemeral pools of water. In addition to the risk of desiccation, tadpoles face predation by their cannibalistic conspecifics, as well from heterospecifics (e.g. Odonate naiads) that occur in these pools (Rojas, 2014; 2015). The larval period lasts approximately 2 months in the wild (Rojas and Pašukonis, 2019) though the laboratory population has had a longer range (2.5-3 months). We used tadpoles from a breeding laboratory population of Dendrobates tinctorius kept at the University of Jyväskylä, Finland. Adult pairs were each housed in a 55L terrarium that contained layered gravel, leaf-litter, moss substrate and was equipped with a shelter, ramps, and live plants. Terraria were maintained at $26 \mathrm{C}( \pm 2 \mathrm{C})$ and were automatically misted with reverse osmosis water four times a day, maintaining a humidity around $95 \%$ and lit with a 12:12 photoperiod. Frogs were fed live Drosophila fruit flies coated in vitamin supplements three times per week. Tadpoles were raised singly in $10 \times 6.5 \times 5 \mathrm{~cm}$ cups which are filled with spring water, and fed an ad libitum diet of fish food (JBL NovoVert flakes) three times a week. Adult and tadpole health and water levels were checked daily, and experimental tadpoles were weighed and photographed weekly. Experiments began in October 2019 and continued through April 2020. This experiment was permitted by the National Animal Experiment Board (ESAVI/9114/04.10.07/2014).

Tags - Visible implant elastomers are a 2-part silicone-based polymer that is injected as a liquid that hardens to a pliable consistency once warmed (VIE; Northwest Marine Technology Inc.).

The result is a small color band on the surface of the animal that can be detected by the naked eye. There is a range of 10 possible colors for application, 6 of which are fluorescent.

Visualization of fluorescent tags can be enhanced using a UV light. VIE tags have been successfully used in diverse taxa across developmental stages (e.g. echinoderms: Martinez et al. 2013; fish: Croft et al., 2003; salamanders: Campbell Grant, 2008). 
162 Anesthesia - Prior to tagging, tadpoles were anesthetized in a $14 \mathrm{~mL}$ solution of a $1 \mu 1: 1 \mathrm{~mL}$ ratio

163 of 2-PHE to spring water. 2-PHE is an oily liquid at room temperature and does not need to be

164 buffered for anesthetic purposes (Coyle et al., 2004). The solution was reused multiple times for

165 multiple tadpoles within a single day of tagging (max. 10 tadpoles tagged each session); its effect

166 did not deteriorate after multiple uses. Each day of tagging a new solution was made. Tadpoles

167 were placed in an anesthetic solution until there was no muscular contraction in response to

168 agitation; this process took approximately 3 minutes. We assumed the anesthetic's potency did

169 not degrade because the latency of its effects remained consistent after being applied to multiple

170 tadpoles. The effect of anesthesia on tadpoles lasted approximately 6 minutes; within 10 minutes

171 individuals had regained full muscular function. The effects of anesthesia were similar across

172 developmental stages (Gosner 24-26). We had no deaths in response to our anesthesia procedure

173 which was applied to a total of 40 individuals across both our pilot study and experimental

174 manipulations.

175

176 Tadpole tagging - We applied VIE tags to early larval stages of $D$. tinctorius and monitored

177 tadpoles across development (Fig 1) to ensure the presence of the tags over time, and to test the

178 effects of larval tagging and tag retention. Previous studies reporting tadpole tagging have been

179 done primarily with late-term tadpoles (Gosner stage 30+) whose snout-vent lengths (SVL) were

180 double or triple the SVL of tadpoles in our experiment (Andis, 2018; Bainbridge et al., 2015;

181 McHarry et al., 2018). Other studies also worked with amphibians who produce large egg

182 clutches (Litoria aurea, 37000 eggs/clutch (Pyke \& White, 2001); American bullfrog, 12000

183 eggs/clutch (Howard, 1978); Alytes obstetricans, 50 eggs/clutch (Reading \& Clarke, 1988)),

184 which allowed for large tag sample sizes $(\mathrm{n}=53-90$, depending on study). Dendrobates

185 tinctorius lay clutches that range from 2 to 5 eggs with a high level of mortality (Rojas \&

186 Pašukonis, 2019). Due to the reproductive limitations of the system, our sample total ( $\mathrm{n}=27$

187 tagged, $\mathrm{n}=11$ control) is less than previously published data.

188

189 Elastomer was mixed and loaded into syringes prior to each tagging session, according to the

190 Northwest Marine Technology VIE tag protocol. Elastomer was stored in a freezer (-20C) during

191 extended periods of disuse and in a refrigerator between individual tagging sessions; we found

192 that mixed elastomer was no longer applicable after a storage period of longer than three

Peer] reviewing PDF | (2020:04:48482:1:2:NEW 2 Jul 2020) 
193 months. Average tagging procedure was executed in under 90 seconds. Throughout our pilot

194 study we found that tag retention was most effective when placed dorsally; thus, this experiment

195 only contained dorsally marked tadpoles. Each tadpole was marked only once.

Immediately after being anesthetized, tadpoles were prepared for tagging. This was done by removing tadpoles from the anesthetic solution and placing them on a laminated surface where they were dried with a paper towel to improve grip. Once excess moisture was removed from the body (without completely drying out the tadpole), a $3 \mathrm{~mL}$ insulin syringe with a $30 \mathrm{G} / 127 \mathrm{~mm}$ needle was placed subcutaneously and dye (approx. $1 \mu \mathrm{l}$ ) was injected. For this experiment, we used a fluorescent green elastomer, though any color tag would have been suitable for application. After tag injection, tadpoles were placed under UV light to ensure proper placement of the tag. Proper placement was qualified as the tag being injected deep enough to not fall out (directly under epidermis) but shallow enough to be visible with the help of a UV light. Tadpoles were then cleaned with spring water and the status of the tag was checked again. Tadpoles posttagging were placed in a pool of spring water and observed for 10 minutes to ensure proper return of muscular function. After the observation period, tadpoles were returned to the pool of water in which they were living

211 Tag observation - Tags were observed once a week by a single person. Tadpoles were placed on 212 a laminated surface, cleaned with spring water, dried, and checked both dorsally and ventrally

213 for the presence of a tag using a UV light. Observers were not blind to the treatment or identity

214 of each tadpole, as the observer both photographed and weighed all experimental tadpoles within 215 a recording session.

217 Statistical analysis

218

219 Tag retention and observation model

220 VIE tag retention and observation was modeled using a Bayesian CJS (Cormack-Jolly-Seber) 221 survival model (see R and JAGS code in supplementary materials; Jolly, 1965; Lebreton et al., 222 1992; Seber, 1965). For each individual, we considered tag observation as a categorical variable 223 that was recorded as absent (0) or present (1); tags that had been lost and not re-observed were 
224 marked NA after the last confirmed observation. We assessed the status of the tag and tadpole 225 development (size, weight) weekly. Tag retention was recorded as present (1) for all weeks 226 previous to the last observation and recorded as NA for all those that followed. Our coding

227 schematic takes into account observer error as a tag that is not observed at one time point but

228 seen later in development is recorded as "retained" throughout the entire unobserved period. The 229 retention status of the tag is unknown after the last positive observation. Distinguishing 230 observation and retention rates allowed us to calculate the rate of false negatives in tag 231 observation. Our model considered weekly discrete time steps where the retention and 232 observation of the tag were considered latent variables that occurred with a certain probability $(\phi$ 233 and $p$, respectively following the nomenclature commonly used for CJS models).

We considered five possible models (M1-5) of increasing complexity for $\phi$ and $p$ : M1 assumed constant probabilities of observation and retention, M2 considered a week effect on both probabilities using a logit link function, M3 took into account both a week effect on $\phi$ and $p$ as well as individual identity as a random effect, M4 had the same parameters as M3, but considered the weight at time of tagging, and M5 had to same parameters of M4 but included individual identity as a random effect. Tag retention and observation were defined as following a binomial distribution with a probability $\phi$ and $p$ respectively for all models. In models M2- M5 retention and observation varied for each week of development $(t)$, thus we used a logit link function to determine $\phi$ and $p$ for each week considered. In model M3 and M5 retention and observation were also influenced by individual identity (id), to account for it, we sampled the random effect parameter estimates from a normal distribution with a certain standard deviation for each individual which were later incorporated to the same logit link function.

For each model we used an MCMC approach considering uninformative priors for all parameters (see supplementary materials) and simulation run characteristics of 4 chains, 100,000 iterations with a 5,000 burn-in and a thinning of 10 . Chain convergence was assessed using a potential scale reduction factor (PSRF) of our parameter estimates which discarded any model run that resulted in a PSRF larger than 1.1 or smaller than 0.9 . We checked sample independence by determining

253 the effective sample size of each parameter.- We did not consider any model run with less than 2545000 independent samples for any parameter. 
256

257

258

259

260

261

262

263

264

265

266

267

268

269

270

271

272

273

274

275

276

277

278

279

280

281

282

283

284

\section{5}

Model selection was based on the lowest DIC value (Deviance Information Criterion) and biological relevance. The most likely discrete probabilities of retention and observation for each week were based on the posterior distributions generated by our model, these values were used to visualize the cumulative probability of tag detection across larval development.

\section{Tadpole growth rates}

Growth rates were compared between treatments using a linear mixed-effect model (LMM). Weekly weight ( $\sim$ weight) and treatment ( $\sim$ treatment) were coded as additive predictors in the growth rate model. Tadpole ID was used as a random effect on the intercept. Growth between treatments was compared by calculating weekly rate changes across development for both treatments, and then rate percent was used in model analysis which were evaluated with a Kenward-Roger's method ANOVA. Growth rate models were chosen as a result of Akaike Information Criterion output (AIC; Akaike, 1973).

\section{Tadpole survival rates}

We used a Kaplan-Meier survival curve to visualize treatment effect on tadpole survival. A mixed effects Cox model was used to calculate the parameters and uncertainty of tagging on survival. Survival object was parameterized with respect to death and time in response to treatment and took individual tadpole identity into account as a random effect (Surv(Week, Dead) $\sim$ Treatment $+(1 \mid$ ID)). Survival was coded as a binomial response (alive (0), dead (1)).

We took a frequentist approach in modeling tadpoles survival rates as it has been suggested that in the absence of reliable informative priors, Cox models are preferable for survival data (Omurlu et al., 2009).

All models and statistics were performed in the program R using base R (v. 3.6.1, R Development Core Team, 2019) with additional packages "survival" (Therneau, 2014), "coxme" (Therneau, 2020), "dplyr" (Wickham et al., 2019), "lme4" (Bates et al., 2015), "pbkrtest" (Halekoh \& Højsgaard, 2014), “JAGS” (Plummer, 2003), and “R2jags” (Su \& Yajima, 2015).

\section{Results}


287 Tag success - Out of our 27 fluorescent tags, $81 \%(22 / 27)$ were successfully detected in

288 tadpoles over the first month of application. This decreased to a little over $50 \%(8 / 15)$ detection

289 by the third month of application, which also marks the approximate time of tadpole

290 metamorphosis. Tags were observed in four out of the 11 tadpoles $(36 \%)$ that survived past

291 metamorphosis. None of our experimental frogs retained their tags after 5 months of

292 development. Mean weight at time of tagging was $0.12 \mathrm{~g}( \pm 0.019 \mathrm{SE})$ for tagged tadpoles and

$2930.099 \mathrm{~g}( \pm 0.015 \mathrm{SE})$ for control tadpoles. Control tadpole weights ranged from 0.0307 to $0.18 \mathrm{~g}$ at

294 initial weigh-in, tagged tadpole weights ranged from 0.0318 to $0.36 \mathrm{~g}$ at time of tagging. The

295 smallest successful tag was applied at $0.0318 \mathrm{~g}$, which was a tadpole who had recently hatched

296 (approximately Gosner stage 25). Our experimental tadpoles were tagged in the early larval

297 stages of development: the youngest successful tag was applied on recently hatched tadpoles

298 who had yet to be transported by their fathers. Tagging did not seem to prevent transport

299 behavior by the father, although we observed transport of tagged tadpoles in only two instances.

300 Tagging at this life stage is especially delicate and requires a practiced hand. We attempted

301 embryonic tagging in pilot studies, but were not able to successfully inject the tag without

302 permanently damaging the embryo.

303

304 The model that had the lowest DIC did not include a week effect or an individual random effect

305 (M1). There was no improvement in model quality when including tadpole mass at time of

306 tagging, suggesting that initial tadpole mass had no effect on tag observation throughout

307 development. It is important to note that tag observation sometimes changed throughout

308 development, and tags that were not observed one week sometimes were detectable later in

309 development (see Fig 2). Instances where tags were not observed could be due to individual

310 growth, resulting in a tag being obstructed by a physical structure (i.e. muscle, tissue) for a

311 period of time. For example, the cumulative probability of tag retention $(\phi)$ until the third month

312 of development was $0.61(0.33-0.80,95 \% \mathrm{CI})$ while the cumulative probability of tag

313 observation $(p)$ was $0.38(0.16-0.59,95 \% \mathrm{CI})$, this demonstrates that after 12 weeks the rate of

314 false negatives is approximately $23 \%$. On average, the difference between cumulative retention

315 and observation rates was about $15 \%$.

316 
317 Growth rate - We found no significant difference in weekly growth rate between control and

318 tagged tadpoles (Fig 3), indicating that tagging does not affect tadpole growth (lmer, ANOVA

319 Kenward-Roger's method, $\mathrm{F}(1,37)=1.12, \mathrm{p}=0.296)$. Weekly tadpole growth rate significantly

320 decreased across time (lmer, ANOVA Kenward-Roger's method, $\mathrm{F}(1,415)=56.4, \mathrm{p}=0.03563^{-}$

$\left.321^{11}\right)$.

322

323 Survival - There was no significant difference in survival between control and tagged groups

324 across larval stages of development. Mortality across the first three months was $18 \%(\mathrm{n}=5 / 27)$

325 for tagged tadpoles and 27\% $(n=3 / 11)$ for control tadpoles (Fig 4). A mixed effects Cox model

326 did not find any significant difference in survival based on treatment (coxme, $z=0.09, p=0.93$ ).

327 Post-metamorphic survival was excluded from analysis due to unnaturally high froglet loss

328 throughout the lab colony which is not indicative of tag impact on froglet survival, but likely

329 ineffective laboratory practices for juvenile health. At time of publication (May 2020) $n=3$

330 tagged tadpoles and $n=1$ control tadpoles were alive.

331

\section{Discussion}

333

334 In our study we applied VIE tags on D. tinctorius tadpoles and monitored them across larval

335 development under laboratory conditions. Compared to previously published visible implant

336 studies, our approach presents application at the youngest developmental stage, and is one of the

337 first studies (after Bainbridge et al., 2015; Warne and Crespi, 2015; Andis, 2018) to follow tags

338 across metamorphosis. To our knowledge, this is the first attempt of larval tagging in a tropical

339 frog, as previous work focused exclusively on species from temperate regions (Bainbridge et al.,

340 2015: Litoria aurea; Courtois et al., 2013, Alytes obstetricans; Nauwelaerts et al., 2000, Rana

341 esculenta). The successful application of VIE tags for the first time in a tropical species with

342 elaborate parental care provides valuable opportunities to investigate parent-offspring

343 interactions and dispersion of these species in a natural context.

344

345 Similar to other studies, we found no difference in growth rate or survival between tagged and

346 control treatments. Based on our weekly weigh-ins and LMM model, we did not detect any

347 significant impact of tagging on growth rates across development (see Fig 3). Throughout our 
348 study, tadpoles grew significantly faster earlier in development which could be due to laboratory

349 conditions (a high-food, no-competition environment). Given the circumstances, tadpoles may

350 have invested energy in growing early in development which would help avoid predation and

351 decrease latency to metamorphosis in the wild (Caldwell \& De Araújo, 1998; Rojas, 2014). We

352 found no effect of tagging on D. tinctorius survival across development; however, we had high

353 rates of post-metamorphic mortality across our laboratory population which impacted our ability

354 to assess tag success across development. Natural history studies of D. tinctorius have shown

355 high larval death rates (Rojas \& Pašukonis, 2019). Although our tadpoles were not subject to the

356 same pressures as wild populations, the mortality we observed across both treatments reflect the

357 precariousness of early life stages in D. tinctorius.

358

359 After the first month of observation, 81\% of tadpoles retained their tag, which is on par with

360 retention rates reported in other tagging efforts (Anholt et al., 1998; Martin, 2011). Other studies

361 report even higher rates of success with tadpole elastomer tags (Courtois et al., 2013 (100\%),

362 Bainbridge et al., $2015(100 \%)$ ) which could be due to a shorter larval period and larger

363 individuals at time of application. Retention rates of this study are of note because VIE tags have

364 been extensively used for mark-recapture studies in fishes and anurans; when taking into account

365 our tag retention rate and our tagging procedure (which takes less than 90 seconds), we can

366 conclude that larval VIE mark-recapture studies on tropical amphibians is feasible.

367

368 A relevant note about implant tagging is that it is limited to observer perception. As tadpoles

369 develop, morphological and phenotypic individual changes can facilitate or mask the presence of

370 a tag. Most importantly, the lack of tag observation should not be assumed to indicate tag loss.

371 In our experiment we were able to differentiate the cumulative probability of retention versus

372 observation over time as a result of weekly checks of tag condition in experimental tadpoles to

373 account for false negatives. Over three months of development tags could go multiple weeks

374 unobserved; finding them later in development indicated that tags were not lost, but had shifted

375 position or been re-exposed as a result of growth. This is important to take into account for mark-

376 recapture studies in settings where regular sampling or capture of the entire tagged population

377 isn't feasible. Our model estimates an average 15\% difference in tag observation versus retention 
378 across larval development which is an error that can be incorporated as an informative prior in

379 future tagging studies.

380

381 VIE tags come in a range of fluorescent colors, making the distinction of clutches or individuals

382 from a distinct cohort possible. This is especially relevant for the larval stages of $D$. tinctorius

383 when tadpoles are aggressive cannibals, as tagging efforts would help distinguish resident

384 tadpoles in phytotelmata. Thus, tagging could be used to help monitor who is being deposited

385 and who is getting attacked, allowing us to track interactions between tadpoles in ephemeral

386 pools (Rojas, 2015). Moreover, VIE tagging of $D$. tinctorius makes it possible to successfully tag

387 tadpoles before they are picked up and transported by their parent. Elastomer tags most clearly

388 fluoresce under low-light conditions, making them ideal for their application in wild D.

389 tinctorius tadpoles which live in dimly lit closed canopy rainforest.

390

391

VIE tags are one of the smallest tagging methods available for field studies. With respect to other tagging methods, VIA tags require a minimum SVL of $2 \mathrm{~cm}$ (Courtois et al., 2013) and PIT tags require $4 \mathrm{~cm}$ (Courtois et al., 2013), making VIE tags a unique option to study larval dynamics. VIE tags are not more than $4 \mathrm{~mm}$ in length, meaning that their successful application presents new opportunities to study larval amphibians that may not have been considered in the past. For example, Anomaglossus beebei, a small endemic poison frog from Guyana, has been seen to transport tadpoles multiple times throughout development (CF, personal observation). Larval tagging of this species could help decipher how shifting male territories influences larval care and transport, and if newly established males take care of tadpoles that are not their own. Early larval tagging could also work for Allobates femoralis, another tadpole transporter, to understand the shifting genetic diversity within phytotelmata across time (Erich et al., 2013).

402

Coupled with the unique patterning of $D$. tincorius that emerges in late metamorphosis and settles in adulthood (Courtois et al., 2012; Rojas \& Endler, 2013), tags can provide early life identification that could be followed by pattern recognition, enabling individual discrimination throughout an individual's entire lifespan. Bainbridge et al. (2015) report recently metamorphosed VIE tag retention to be high (88-95\%); we also find that tags that lasted 
409 from implant tagging, genetic tracking has proven to be a reliable method to follow amphibian

410 larvae throughout development into adulthood. With this said, genetic tracking does not provide

411 immediate individual detection; further, studies using this method have been limited to

412 individuals in a closed population, making the recapture of (surviving) tracked individuals

413 reasonably certain (Ringler et al., 2015). In D. tinctorius, however, males can travel remarkable

414 distances while carrying tadpoles (Pašukonis et al., 2019) making genetic tracking a less suitable

415 method for individual distinction in this species. Andis (2018) also did important work dyeing

416 tadpoles of Rana sylvatica with calcein. This dye appears to persist across metamorphosis,

417 though it should be noted that their development is much shorter than D. tinctorius and staining

418 only allows for presence/absence detection. The presence of a VIE tag (and the range of colors

419 available for application) allows for immediate discrimination of multiple groups/cohorts which

420 may be an important advantage when conducting behavioral experiments and elucidating natural

421 history dynamics in the wild.

422

423 Our study presents a successful continuation expanding marking methodology to larval tropical

424 species. Using laboratory conditions, we were able to mimic a common scenario where

425 experimental tadpoles were left to develop in small pools of water. This is reflective of the most

426 common parental behavior exhibited by D. tinctorius, where males transport newly-hatched

427 tadpoles to develop in small water holdings (Rojas \& Pašukonis, 2019). Future studies in field

428 conditions would be useful to supplement these findings. For example, it will be important to

429 understand how tadpole interaction with conspecifics, heterospecifics, and predators affects tag

430 retention. It is also important to consider how tagging will affect individual behavior and

431 success. Although different from VIEs, studies have found tadpole staining to have effects both

432 on predator response (Carlson and Langkilde, 2012) and aggression levels (Fischer et al., 2020);

433 it is important to acknowledge that manipulating animals can entail unexpected/unintended

434 consequences and that further studies working with tags in natural settings are warranted.

435 However, based on previously published data and the observation rates of our elastomers in this

436 experiment, we believe that the application of elastomers in the wild is already an appropriate

437 method to distinguish tadpoles for behavioral experiments. Elastomers are small, successful, and

438 relatively easy to apply in early amphibian life stages. Our study contributes to the growing body 
439 of methods-based research demonstrating that visible implant elastomers are a viable tagging

440 solution on a variety of anuran species in early development.

441

\section{Conclusions}

443

444

Differentiating individuals/cohorts can be a powerful tool when conducting behavioral experiments. Often, marking animals is a technique used to distinguish individuals when physical features are not distinct enough for visual differentiation. Choosing an optimal tag for a system is a tradeoff between reliability and invasiveness and is often limited to product cost and efficiency in identification. Elastomers (VIE) are injectable polymers that have been extensively used in fish and anuran systems. However, until this point, they have been applied to large larvae or adults and have been heavily biased towards common, temperate species. Here, we present the first application of VIE tags on a small larval tropical frog (Dendrobates tinctorius) and follow tag success across development. We found that (1) VIE tags can be successfully applied to recently hatched tadpoles, (2) tags can be reliably followed throughout larval development and sometimes retained across metamorphosis, and (3) VIE tags do not appear to interfere with parental care behavior (i.e. tadpole transport). Our study expands the application of tagging to early developmental stages in tropical amphibians which can be of use in behavior, conservation, and natural history research studies in the future.

\section{Funding}

460

461 Funding for this project was provided by BR, who is currently funded by the Academy of

462 Finland (Academy Research Fellowship, Project No. 21000042021).

463

\section{Acknowledgements}

465

We would like to thank German Orizaola, Andis Arietta, Max Lambert, Janne Valkonen, Lutz Fromhage, and one anonymous reviewer for such constructive and supportive comments. We also owe an enormous thank you to Matthieu Bruneaux for his conversation and 
470 Kumpulainen and Emmi Alanen, and lab technician Teemu Tuomaala for helping with tadpole 471 care-- it takes a village.

472

\section{References}

474 Acme-Hardestry. (2013). Phenoxyethanol ; 2-phenoxyethanol. Federal Register, 77(58), 1-6.

475 Akaike, H. (1973). Maximum likelihood identification of Gaussian autoregressive moving 476 average models. Biometrika, 60(2), 255-265.

477 Andis, A. Z. (2018). A new, noninvasive method of batch-marking amphibians across

478 developmental stages. Herpetological Conservation and Biology, May.

479 Anholt, B. R., Negovetic, S., \& Som, C. (1998). Methods for anaesthetizing and marking larval $480 \quad$ anurans. Herpetological Review, 29(3), 153-154.

481 Bailey, L. L. (2004). Evaluating Elastomer Marking and Photo Identification Methods for 482 Terrestrial Salamanders: Marking Effects and Observer Bias. Herpetological Review, 35(1), $483 \quad 38-41$.

484 Bainbridge, L., Stockwell, M., Valdez, J., Klop-Toker, K., Clulow, S., Clulow, J., \& Mahony, M. 485 (2015). Tagging tadpoles: Retention rates and impacts of visible implant elastomer (VIE) 486 tags from the larval to adult amphibian stages. Herpetological Journal, 25(3), 133-140.

487 Bates, D., Mächler, M., Bolker, B. M., \& Walker, S. C. (2015). Fitting linear mixed-effects

488 models using lme4. Journal of Statistical Software, 67(1).

489 https://doi.org/10.18637/jss.v067.i01

490 Caballero-Gini, A., Villafañe, D. B., Romero, L., Ferreira, M., Cañete, L., Laino, R., \&

491 Musalem, K. (2019). Visible implant alphanumeric (VIA) as a marking method in the lesser 492 snouted treefrog scinax nasicus. Acta Herpetologica, 14(2), 129-133.

493 https://doi.org/10.13128/a_h-7751

494 Caldwell, J. P., \& De Araújo, M. C. (1998). Cannibalistic interactions resulting from

495 indiscriminate predatory behavior in tadpoles of poison frogs (Anura: Dendrobatidae).

496 Biotropica. https://doi.org/10.1111/j.1744-7429.1998.tb00372.x

497 Campbell Grant, E. H. (2008). Visual Implant Elastomer Mark Retention Through

498 Metamorphosis in Amphibian Larvae. Journal of Wildlife Management, 72(5), 1247-1252.

499 https://doi.org/10.2193/2007-183

500 Courtois, E. A., Lelong, C., Calvez, O., Loyau, A., \& Schmeller, D. S. (2013). The use of visible 
501 implant alpha tags for anuran tadpoles. Herpetological Review, 44(2), 230-233.

502 Courtois, E. A., Pineau, K., Villette, B., Schmeller, D. S., \& Gaucher, P. (2012). Population

503 estimates of Dendrobates tinctorius (anura: Dendrobatidae) at three sites in French Guiana

504 and first record of chytrid infection. Phyllomedusa, 11(1), 63-70.

505 Coyle, S. D., Durborow, R. M., \& Tidwell, J. H. (2004). Anesthetics in Aquaculture. SRAC

506 Publication, 3900(3900). http://fisheries.tamu.edu/files/2013/09/SRAC-Publication-No.-

507 3900-Anesthetics-in-Aquaculture.pdf

508 Denoel, M., \& Joly, P. (2000). Neoteny and progenesis as two heterochronic processes involved 509 in paedomorphosis in Triturus alpestris (Amphibia: Caudata). Proceedings of the Royal

$510 \quad$ Society B: Biological Sciences, 267(1451), 1481-1485.

511 https://doi.org/10.1098/rspb.2000.1168

512 Dugas, M. B., Stynoski, J., \& Strickler, S. A. (2016). Larval aggression is independent of food

513 limitation in nurseries of a poison frog. Behavioral Ecology and Sociobiology, 70(8), 1389-

514 1395. https://doi.org/10.1007/s00265-016-2148-5

515 Erich, M., Ringler, M., Hödl, W., \& Ringler, E. (2013). Bet-hedging in Tadpole Deposition in 516 the Neotropical Frog. Universität Wien.

517 Faragher, S. G., \& Jaeger, R. G. (1998). Tadpole bullies: Examining mechanisms of competition 518 in a community of larval anurans. Canadian Journal of Zoology, 76(1), 144-153.

519 https://doi.org/10.1139/z97-177

520 Gonzalez, S. C., Touchon, J. C., \& Vonesh, J. R. (2011). Interactions between competition and 521 predation shape early growth and survival of two neotropical hylid tadpoles. Biotropica, 522 43(5), 633-639. https://doi.org/10.1111/j.1744-7429.2010.00748.x

523 Halekoh, U., \& Højsgaard, S. (2014). A Kenward-Roger approximation and parametric bootstrap 524 methods for tests in linear mixed models-the R package pbkrtest. Journal of Statistical $525 \quad$ Software, 59(9), 1-32. https://doi.org/10.18637/jss.v059.i09

526 Howard, R. D. (1978). The Evolution of Mating Strategies in Bullfrogs, Rana catesbeiana.

527 Evolution, 32(4), 850. https://doi.org/10.2307/2407499

528 Jolly, G. M. (1965). Explicit Estimates from Capture-Recapture Data with Both Death and 529 Immigration- Stochastic Model. Biometrika, 52(1), 225-247.

530 Lebreton, J.-D., Burnham, K. P., Clobert, J., \& Anderson, D. R. (1992). Modeling Survival and 531 Testing Biological Hypotheses Using Marked Animals : A Unified Approach with Case 
532

533

534

535

536

537

538

539

540

541

542

543

544

545

546

547

548

549

550

551

552

553

554

555

556

557

558

559

560

561

562

Studies. Ecological Society of America, 62(1), 67-118.

Martin, R. A. (2011). Evaltuating a novel technique for individual identification of anuran tadpoles using coded wire tags. Herpetological Conservation and Biology, 5(2), 253-262. https://doi.org/10.1227/01.NEU.0000125007.03145.00

McHarry, K. W., Abbott, J. M., van Hattem, M. G., \& Hudgens, B. R. (2018). Efficacy of visible implant elastomer tags with photographic assist for identifying individuals in capture-markrecapture studies using larval frogs. Herpetological Conservation and Biology, 13(3), 576585.

Ménard, N., Segesser, F. Von, Scheffrahn, W., \& Pastorini, J. (2001). Is male - infant caretaking related to paternity and / or mating activities in wild Barbary macaques ( Macaca sylvanus )? Life Sciences, 324, 601-610.

National Center for Biotechnology Information. (2020). 2-Phenoxyethanol. PubChem Database, $1-48$.

Nauwelaerts, S., Coeck, J., \& Aerts, P. (2000). Visible implant elastomers as a method for marking adult anurans. Herpetological Review, 31(3), 154-155.

Omurlu, I. K., Ozdamar, K., \& Ture, M. (2009). Comparison of Bayesian survival analysis and Cox regression analysis in simulated and breast cancer data sets. Expert Systems with Applications, 36(8), 11341-11346. https://doi.org/10.1016/j.eswa.2009.03.058

Pašukonis, A., Loretto, M.-C., \& Rojas, B. (2019). How far do tadpoles travel in the rainforest? Parent-assisted dispersal in poison frogs. Evolutionary Ecology, 0123456789. https://doi.org/10.1007/s10682-019-09994-z

Perret, N., \& Joly, P. (2002). Impacts of Tattooing and Pit-Tagging on Survival and Fecundity in the Alpine Newt (Triturus Alpestris). Herpetologica, 58(1), 131-138. https://doi.org/10.1655/0018-0831(2002)058[0131:iotapo]2.0.co;2

Pfennig, D. W., Loeb, M. L. G., \& Collins, J. P. (1991). Pathogens as a factor limiting the spread of cannibalism in tiger salamanders. Oecologia, 88(2), 161-166. https://doi.org/10.1007/BF00320806

Phillott, A. D., Skerratt, L. F., McDonald, K. R., Lemckert, F. L., Hines, H. B., Clarke, J. M., Alford, R. A., \& Speare, R. (2007). Toe-clipping as an acceptable method of identifying individual anurans in mark recapture studies. Herpetological Review, 38(3), 305-308.

Plummer, M. (2003). JAGS: A program for Analysis of Bayesian Graphical Models Using Gibbs 
Pyke, G. H., \& White, A. W. (2001). A review of the biology of the Green and Golden Bell Frog Litoria aurea. Australian Zoologist, 31(4), 563-598. https://doi.org/10.7882/AZ.2001.003

Reading, C. J., \& Clarke, R. T. (1988). Multiple clutches, egg mortality and mate choice in the mid-wife toad, Alytes obstetricans. Amphibia Reptilia, 9(4), 357-364. https://doi.org/10.1163/156853888X00035

Ringler, E., Mangione, R., \& Ringler, M. (2015). Where have all the tadpoles gone? Individual genetic tracking of amphibian larvae until adulthood. Molecular Ecology Resources, 15(4), 737-746. https://doi.org/10.1111/1755-0998.12345

573

Ringler, E., Pašukonis, A., Hödl, W., \& Ringler, M. (2013). Tadpole transport logistics in a Neotropical poison frog: indications for strategic planning and adaptive plasticity in anuran parental care. Frontiers in Zoology, 10(1), 67. https://doi.org/10.1186/1742-9994-10-67

Rojas, B. (2014). Strange parental decisions: Fathers of the dyeing poison frog deposit their tadpoles in pools occupied by large cannibals. Behavioral Ecology and Sociobiology, 68(4), 551-559. https://doi.org/10.1007/s00265-013-1670-y

Rojas, B. (2015). Mind the gap : treefalls as drivers of parental trade-offs. Ecology and Evolution. https://doi.org/10.1002/ece3.1648

Rojas, B., \& Endler, J. A. (2013). Sexual dimorphism and intra-populational colour pattern variation in the aposematic frog Dendrobates tinctorius. Evolutionary Ecology, 27(4), 739753. https://doi.org/10.1007/s10682-013-9640-4

Rojas, B., \& Pašukonis, A. (2019). From Habitat Use to Social Behavior : Natural History of a Voiceless Poison Frog, Dendrobates tinctorius. PeerJ. https://doi.org/10.1101/515122

Schulte, L. M., \& Mayer, M. (2017). Poison frog tadpoles seek parental transportation to escape their cannibalistic siblings. Journal of Zoology, 303(2), 83-89. https://doi.org/10.1111/jzo.12472

Seber, G. A. F. (1965). A Note on the Multiple-Recapture Census. Biometrika, 52(1), 249-259.

Su, U., \& Yajima, M. (2015). R2jags: Using R to run “JAGS.” R Packages, 12. https://doi.org/http://cran.r-project.org/package=R2jags

Summers, K., \& Tumulty, J. (2013). Parental Care, Sexual Selection, and Mating Systems in Neotropical Poison Frogs. In Sexual Selection: Perspectives and Models from the Neotropics (pp. 289-320). https://doi.org/10.1016/B978-0-12-416028-6.00011-6 
594 Therneau, T. M. (2014). Package 'survival.'

595 Therneau, T. M. (2020). Package 'coxme.'

596 Touchon, J. C., Urbina, J., \& Warkentin, K. M. (2011). Habitat-specific constraints on induced

597 hatching in a treefrog with reproductive mode plasticity. Behavioral Ecology, 22(1), 169598 175. https://doi.org/10.1093/beheco/arq192

599 Wickham, H., Averick, M., Bryan, J., Chang, W., McGowan, L., François, R., Grolemund, G., 600 Hayes, A., Henry, L., Hester, J., Kuhn, M., Pedersen, T., Miller, E., Bache, S., Müller, K., 601 Ooms, J., Robinson, D., Seidel, D., Spinu, V., ... Yutani, H. (2019). Welcome to the 602 Tidyverse. Journal of Open Source Software, 4(43), 1686.

603 https://doi.org/10.21105/joss.01686

604 
Figure 1

Fluorescent green VIE tag inserted dorsally on Dendrobates tinctorius.

Tag shown on the same individual as (A) a late stage larva, (B) a metamorph, and (C) a recently metamorphosed juvenile. All photos taken with Nikon DS5300 DSLR on $1 \times 1 \mathrm{~mm}$ background under UV light to enhance tag detection.

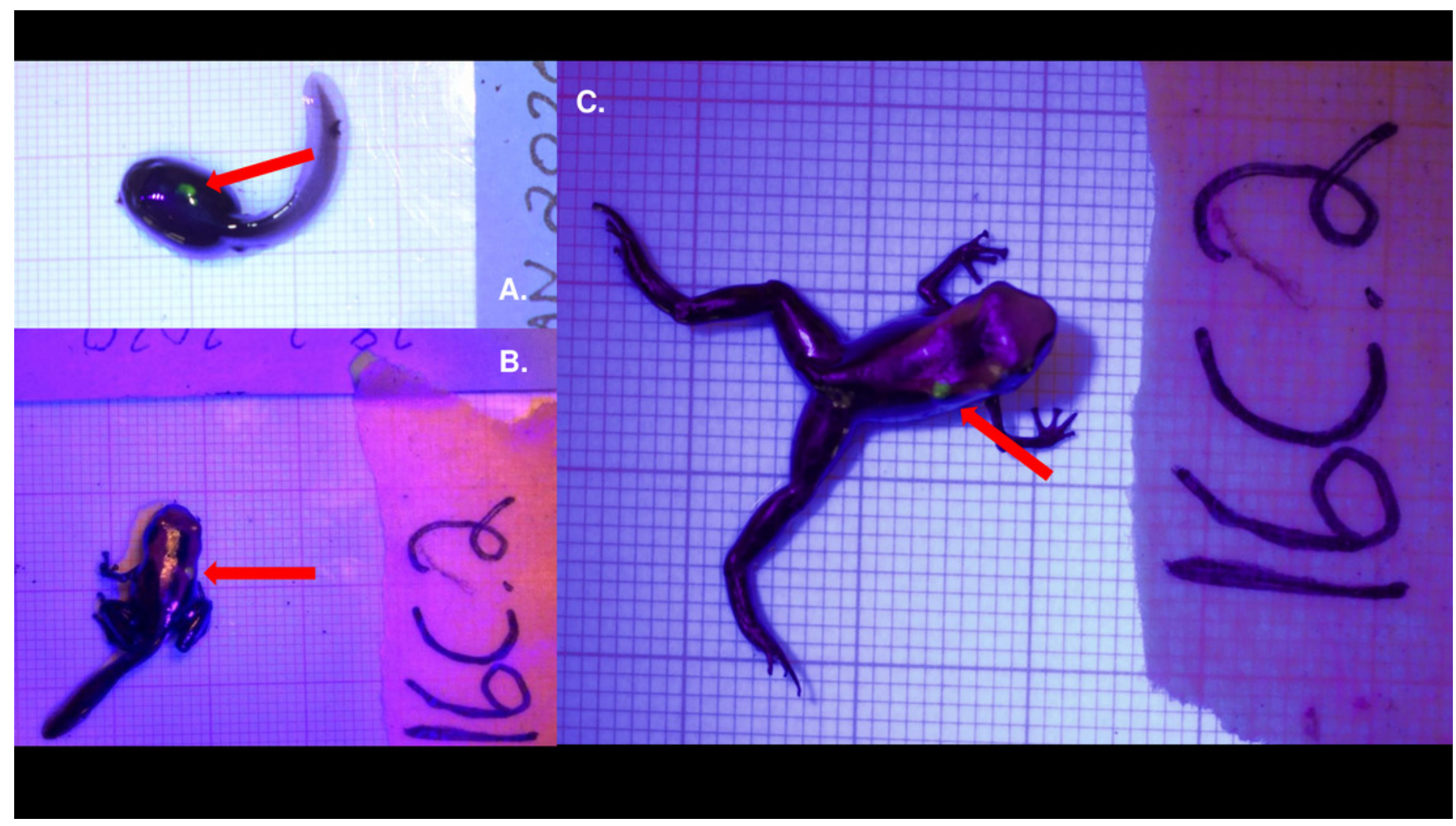




\section{Figure 2}

Estimate of the cumulative probability of tag detection across larval development for model M1.

(A) Probability of tag retention across larval development. Grey points are the probability of tag retention (phi). (B) Probability of tag observation across larval development. Green points are the probability of tag observation $(p)$. All points are posterior means at discrete time intervals corresponding to weeks of development. Grey polygons delimited by black dashed lines indicate the $95 \%$ credible intervals.

A.

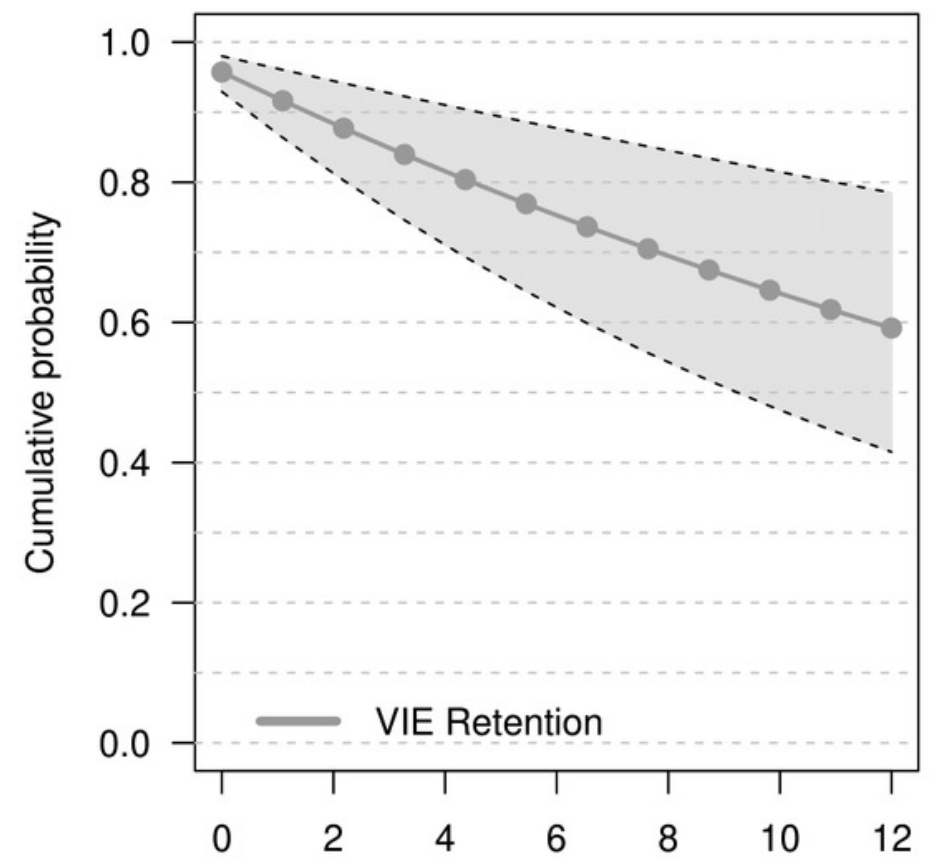

Weeks of development
B.

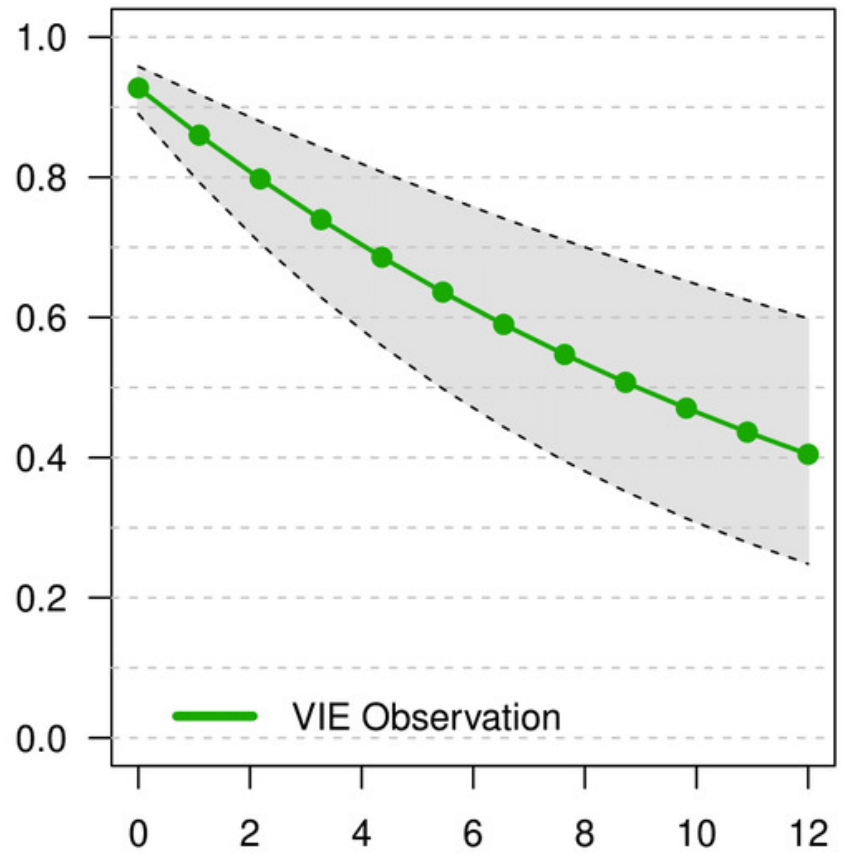

Weeks of development 
Figure 3

Average growth rate of VIE tagged and control group tadpoles.

Diamonds represent the LS mean for which error bars indicate $95 \%$ confidence intervals.

Means sharing letters are not significantly different (Tukey-adjusted comparisons, ANOVA Kenward-Roger's method, $F(1,37.97)=1.12, p=0.296)$.

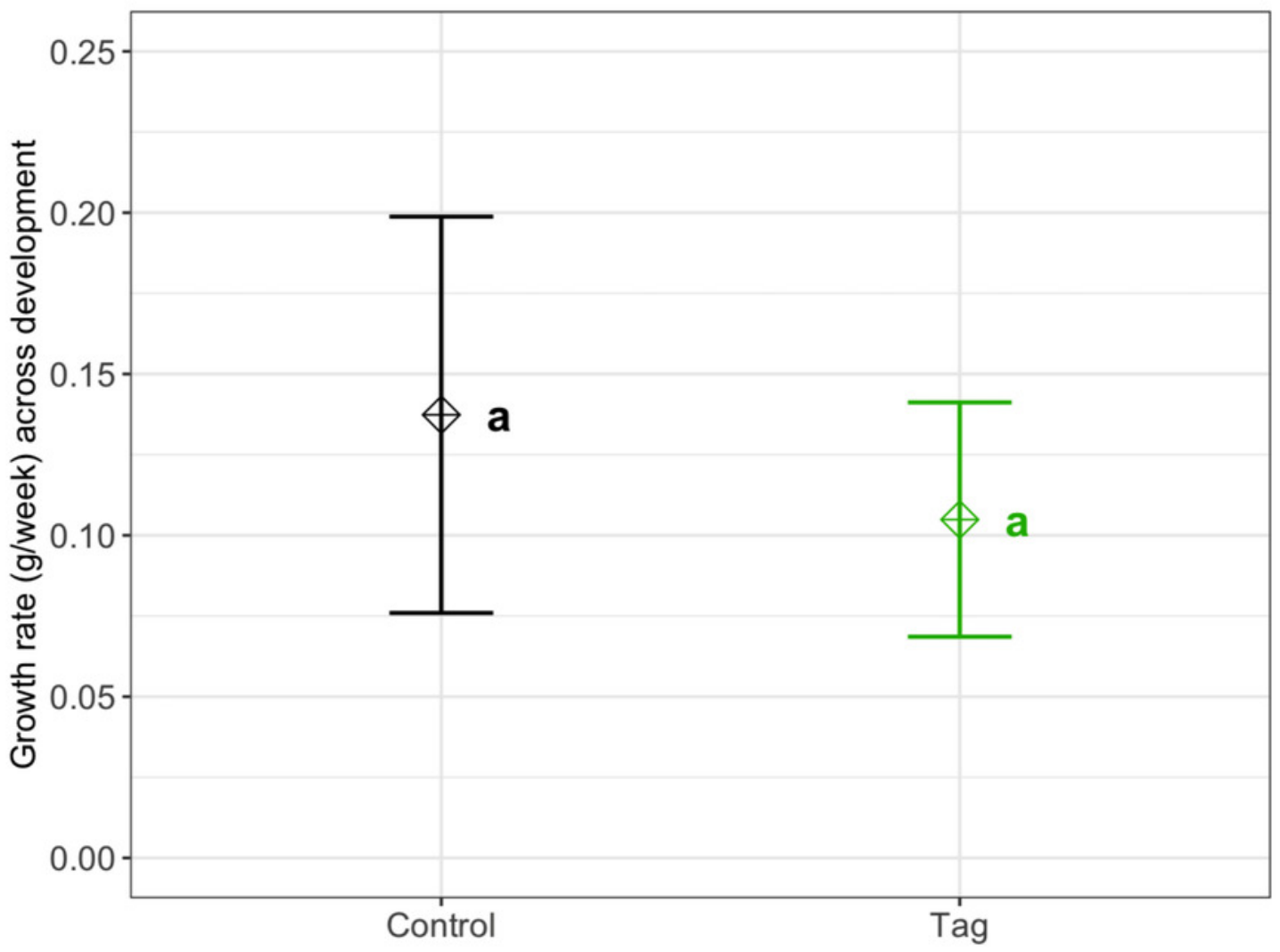




\section{Figure 4}

Kaplan-Meier survival curves of experimental tadpoles across larval development.

Curves represent the probability of survival of tagged and control tadpoles over time. A mixed effects Cox model did not find any significant difference of treatment on tadpole survival across development $(z=0.09, p=0.93)$. Shaded regions represent $95 \%$ confidence intervals. 
Control -+ Tag

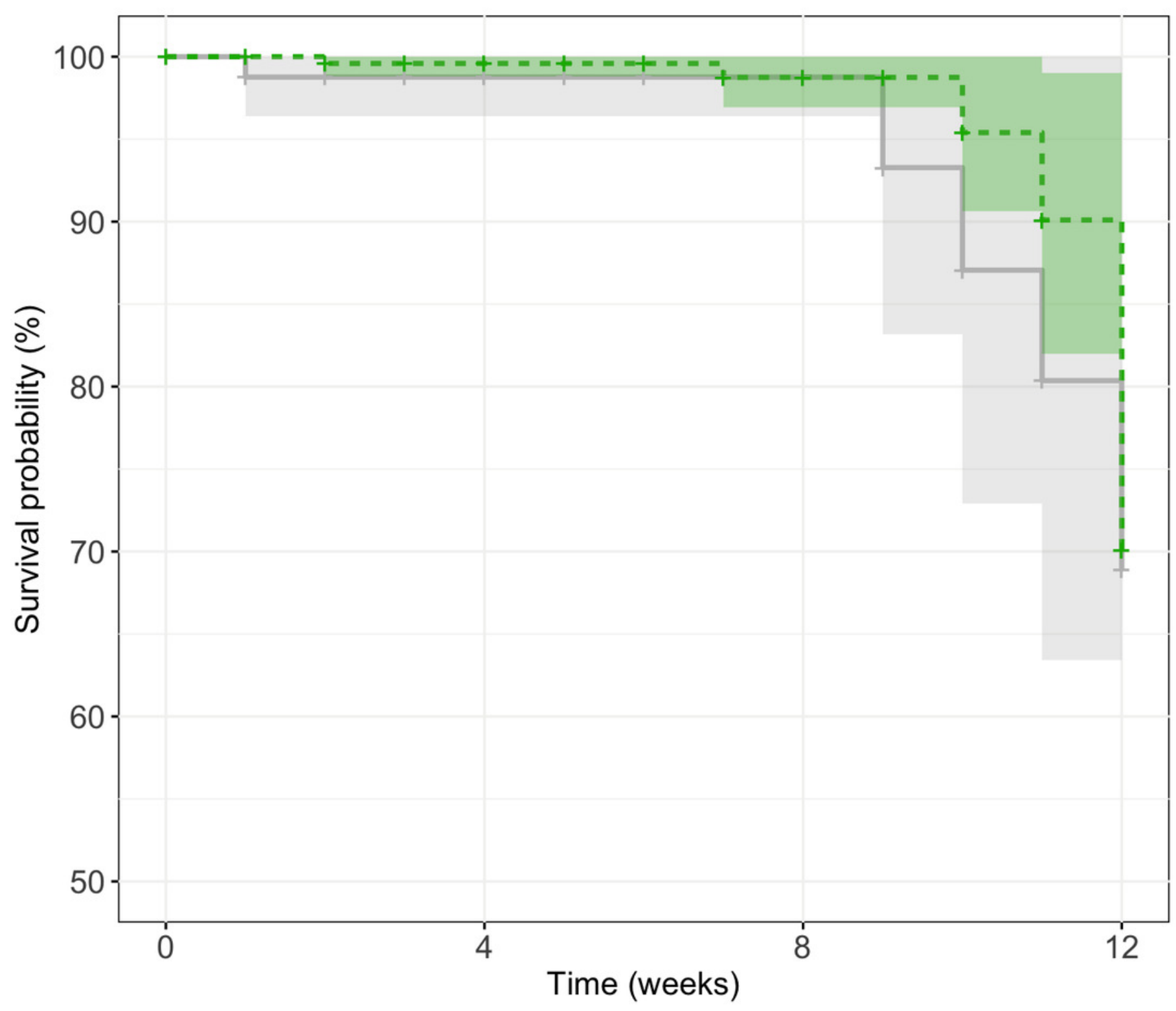

